\title{
COVID-19 Serology Control Panel Using the Dried-Tube Specimen Method
}

\author{
William J. Windsor, ${ }^{1}$ Vijaya Knight, ${ }^{2}$ Patricia A. Merkel, ${ }^{2}$ Molly M. Lamb, ${ }^{1}$ Heidi R. Tucker, ${ }^{3}$ Kyle Carson, ${ }^{3}$ Kelly M. Howard, ${ }^{3}$ \\ Jennifer L. Yates, ${ }^{3,4}$ Mario L. Santiago, ${ }^{5}$ Mary K. McCarthy, ${ }^{5}$ Thomas E. Morrison, ${ }^{5}$ Ross M. Kedl, ${ }^{5}$ Ashley Frazer-Abel, ${ }^{5}$ \\ Kejun Guo, ${ }^{5}$ Gillian Andersen, ${ }^{5}$ Leah Huey, ${ }^{2}$ Bradley S. Barrett, ${ }^{5}$ Jessica M. Colón-Franco, ${ }^{6}$ William T. Lee, ${ }^{3,4}$ and May C. Chu ${ }^{1 *}$ \\ ${ }^{1}$ Colorado School of Public Health, Anschutz Medical Campus, Aurora, Colorado; ${ }^{2}$ Children's Hospital Colorado, Anschutz Medical Campus, \\ Aurora, Colorado; ${ }^{3}$ Division of Infectious Diseases, Wadsworth Center, New York State Department of Health, Albany, New York; ${ }^{4}$ The School of \\ Public Health, The University at Albany, Albany, New York; ${ }^{5}$ University of Colorado Anschutz Medical Campus, Aurora, Colorado; ${ }^{6}$ Cleveland \\ Clinic, Cleveland, Ohio
}

\begin{abstract}
The dried-tube specimen (DTS) procedure was used to develop the COVID-19 serology control panel (CSCP). The DTS offers the benefit of shipping materials without a cold chain, allowing for greater access without deterioration of material integrity. Samples in the panel were sourced from COVID-19 convalescent persons from March to May 2020. The immunoglobulin subtypes (total Ig, IgM, and IgG) and their respective reactivity to severe acute respiratory syndrome coronavirus 2 nucleocapsid, spike, and receptor-binding domain antigens of the samples were delineated and compared with the WHO International Standard to elucidate the exact binding antibody units of each CSCP sample and ensure the CSCP provides adequate reactivity for different types of serological test platforms. We distribute the CSCP as a kit with five coded tubes to laboratories around the world to be used to compare test kits for external quality assurance, for harmonizing laboratory testing, and for use as training materials for laboratory workers.
\end{abstract}

A standardized panel composed of well-characterized plasma/serum specimens can bridge serosurveillance studies, compare test kits, serve as external quality assurance, harmonize tests that measure vaccine efficacy, be used as a training tool for laboratory workers, and be used for postmarket monitoring. The standardized panel can be shared by a global network to link studies and enable inclusive analysis for a variety of use cases, as mentioned, and, more importantly, a standardized control panel can provide longterm quality performance monitoring as reagents and production batches change. We have established a COVID-19 serology control panel (CSCP) using the dried-tube specimen (DTS) protocol ${ }^{1}$ so that the panel can be shipped globally without a cold chain, thus allowing greater access to materials in all resource settings while maintaining sample integrity. Identifying the appropriate test kit for a use case has been made more complicated with more than 120 severe acute respiratory syndrome coronavirus 2 (SARSCoV-2) serological test kits listed by the U.S. Food and Drug Administration under Emergency Use Authorized and/or registered with the Conformitè Européenne-marked European market. The SeroTracker ${ }^{2}$ list shows there are as many research use-only tests being used in clinics and research laboratories. With this unprecedented number of serological testing platforms and algorithms, it is imperative that we prioritize the quality calibration of test kits and platforms to ensure results are meaningful and can be compared across the hundreds of seroprevalence studies being undertaken. ${ }^{3}$ Calibration is especially important for testing in low-resource settings, where immunological testing is more likely to be used than other diagnostic test formats.

Nine highly reactive COVID-19 convalescent plasma samples collected between March and May 2020 by the Vitalant Research Institute (San Francisco, CA) were selected. The selected samples had a neutralization reactivity range of

*Address correspondence to May C. Chu, Colorado School of Public Health, Anschutz Medical Campus, Aurora, CO. E-mail: may.chu@cuanschutz.edu
1:640 to $>1: 10,240$ using a SARS-CoV-2 spike (S) reporter viral particle neutralization assay, and reactivity was confirmed using the Ortho VITROS Anti-SARS-CoV-2 Total immunoglobulin assay (S subunit protein; Ortho-Clinical Diagnostics, Inc. Rochester, NY) (Table 1). ${ }^{4}$ These nine COVID-19 convalescent plasma samples and one pre-2019 human plasma sample were certified to be blood borne and pathogen free by the Vitalant Research Institute. This study was conducted under a University of Colorado-Denver (CU) Human Subjects Research Waiver (protocol 20-0711).

The samples were evaluated by CU Laboratories to determine each sample's reactivity to the $S$, nucleocapsid $(\mathrm{N})$, and receptor-binding domain (RBD) with five SARS-CoV-2 serology methods (Table 1 ). ${ }^{5-8}$ We pooled three samples that represented the highest reactivity to $S, N$, and RBD in a 1:1:1 ratio. The undiluted pool served as the high-reactive (HR) sample and the pre-2019 plasma served as the nonreactive (NR) sample in all assays. The low-reactive (LR) pool was prepared as a 1:4 dilution of the HR using the NR sample as the diluent. We included duplicate LR samples in the CSCP to provide insights into serology assay limits of detection. Then, the three samples-HR, LR, and NR-were evaluated by $\mathrm{CU}$ Laboratories pre- and post-drying.

According to the DTS protocol, ${ }^{1}$ we mixed in $0.1 \%$ green food dye for better visualization. We aliquoted $20 \mu \mathrm{L}$ in a 2-mL Sarstedt vial (NC9180825; Fisher Scientific, USA) and then left the open tube to dry overnight in a high-efficiency particulate air-filtered laminar flow hood. The tubes were capped and stored at $4^{\circ} \mathrm{C}$ during the CSCP kit assembly process, and stored at $-20^{\circ} \mathrm{C}$ afterward to preserve the integrity of the samples for longer term storage. To rehydrate, a DTS vial was rehydrated with $200 \mu \mathrm{L}$ phosphatebuffered saline (PBS) with $0.2 \%$ Tween (PBS diluent), then was allowed to solubilize overnight at $4^{\circ} \mathrm{C}$ before use.

The long-term temperature stability of the dried materials was determined by storing CSCP kits continuously at $-20^{\circ} \mathrm{C}$, $4^{\circ} \mathrm{C}, 25^{\circ} \mathrm{C}, 37^{\circ} \mathrm{C}$, and $45^{\circ} \mathrm{C}$ for 1 week, 2 weeks, 1 month, 3 months, 6 months, and 1 year. The optimal stability of the CSCP for up to 1 year is between $-20^{\circ} \mathrm{C}$ and $25^{\circ} \mathrm{C}$, with loss 
TABLE 1

Test platforms used to characterize the COVID-19 serology control panel samples

\begin{tabular}{|c|c|c|c|c|c|}
\hline Antigens & Antibody type & Format & Test output & Source & Details \\
\hline \multicolumn{6}{|c|}{ Individual donor plasma characterization } \\
\hline \multirow[t]{2}{*}{ S1 } & Total lg & $\begin{array}{l}\text { Chemiluminescent } \\
\text { immunoassay }\end{array}$ & Qualitative & Ortho Vitros Cov2T & EUA \\
\hline & Total lg & $\begin{array}{l}\text { Pseudo-type VSV reporter } \\
\text { neutralization }\end{array}$ & Quantitative & VRI & $\begin{array}{l}\text { RUO; results reported as } \\
\text { NT50 }\end{array}$ \\
\hline \multicolumn{6}{|c|}{ Individual and pooled donor plasma characterization } \\
\hline S1 & Total lg & $\begin{array}{l}\text { Pseudo-type HIV reporter } \\
\text { neutralization }\end{array}$ & Quantitative & CU Anschutz & $\begin{array}{l}\text { RUO; results reports as } \\
\text { NT }\end{array}$ \\
\hline \multirow[t]{2}{*}{ RBD and $\mathrm{N}$} & $\lg G$ & ELISA & Qualitative & CU Anschutz & RUO \\
\hline & $\lg G$ & $\begin{array}{l}\text { Multiplex microsphere } \\
\text { immunoassay }\end{array}$ & Quantitative & CU Anschutz & RUO \\
\hline $\mathrm{N}$ & $\lg M$ and $\lg G$ & ELISA & Qualitative & Epitope & EUA \\
\hline Virus & Total Ig & $\begin{array}{l}\text { Focus reduction } \\
\text { neutralization titer }\end{array}$ & Quantitative & CU Anschutz & $\begin{array}{l}\text { Vero E6 cells cultured } \\
\text { with SARS-CoV-2 USA- } \\
\text { WA1/2020 strain }\end{array}$ \\
\hline
\end{tabular}

$\mathrm{CU}=$ University of Colorado; EUA = U.S. Food and Drug Administration Emergency Use Authorization; N = nucleocapsid proteins; NT = neutralization test; NT50 = neutralization test reported as $50 \%$ of reduction of virus replication; RBD = receptor binding protein; RUO = research use only; S1 = severe acute respiratory syndrome coronavirus 2 spike $1 ;$ SARS-CoV-2 = severe acute respiratory syndrome coronavirus 2; VRI = Vitalant Research Institute; VSV = vesicular stomatitis virus.

of reactivity after 2 months at $37^{\circ} \mathrm{C}$ and non-reactivity at $45^{\circ} \mathrm{C}$ (data not shown).

The CSCP was further characterized and measured against the WHO International Standard (WHO IS). ${ }^{10} \mathrm{We}$ used the microbead immunoassay ${ }^{9}$ for this characterization, and analyzed results by parallel line assay (PLA). The immunoassay reagent preparation protocol and the derivation of the PLA analysis are provided in the Supplemental Materials.

PLA is the standard approach used to convert any analyte to binding antibody units (BAUs) against a known concentration of an analyte standard, represented here as the WHO IS $\mathrm{N}, \mathrm{S}$, and RBD-specific Ig. IgM and IgG were chosen as the analytes to convert HR and LR samples to BAUs. The WHO IS was set to $1,000 \mathrm{BAU} / \mathrm{mL}$ for each antigen-isotype combination, and the CSCP DTS HR and LR samples were considered unknowns. A robust PLA is dependent on the linearity of the dilution curves for both the standard and unknown. Therefore, the dilution curves of all analytes were linearized using a logit transformation on the raw data for both the CSCP DTS standard and WHO IS, measured as median fluorescence intensity. The logit transformation resulted in six to nine serial dilutions within the parallel linear range for comparison of all analytes except $\mathrm{N}$-specific IgM (Figure 1). The Cls for these calculations were less than $25 \%$ (Table 2). However, we were unable to predict a potency for $\mathrm{N}$-specific IgM because of a large difference between the slopes of the WHO IS and CSCP CTS standard for this analyte. Although detectable, the levels of $\mathrm{N}$-specific IgM were
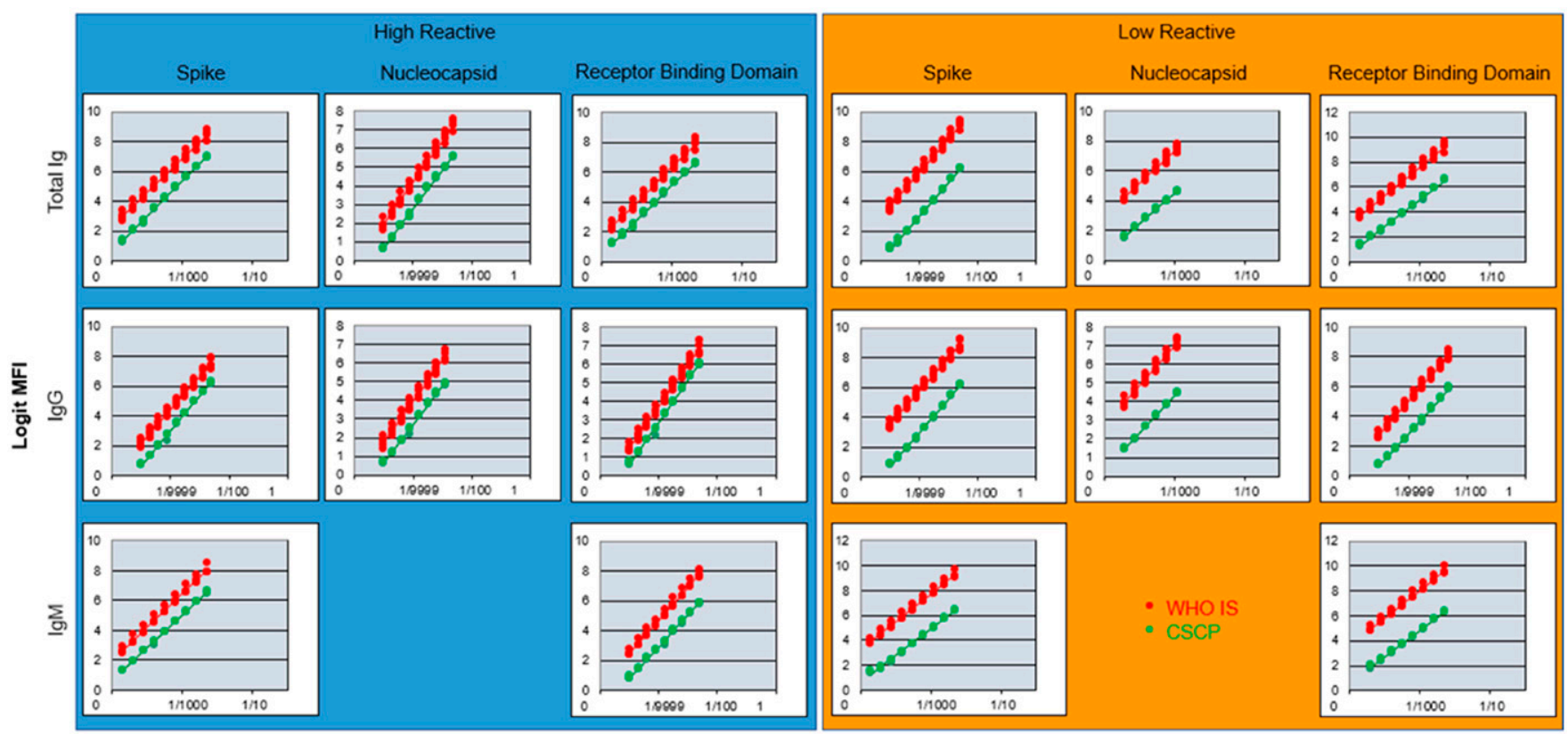

FIGURE 1. Parallel line assay results of COVID-19 serology control panels (CSCPs) compared with the WHO International Standard (WHO IS). Parallel lines obtained by plotting logit-transformed median fluorescence intensity (MFI) for the dilution ranges used to calculate binding antibody units per milliliter of total antibody (lg), lgG, and lgM against the monomeric full-length spike, nucleocapsid, and receptor-binding domain of severe acute respiratory syndrome coronavirus 2 in the high-reactive and low-reactive CSCP reconstituted dried-tube specimen. The WHO severe acute respiratory syndrome coronavirus 2 serology international standard is in red and the CSCP specimen is in green. This figure appears in color at www.ajtmh.org. 
TABLE 2

Potency of COVID-19 serology control panel standards in international binding antibody units

\begin{tabular}{|c|c|c|c|}
\hline & High-reactive sample & Low-reactive sample 1 & Low-reactive sample 2 \\
\hline \multicolumn{4}{|l|}{$\lg$} \\
\hline Nucleocapsid proteins & $212.1(185.3-241.9)^{*}$ & $49.7(38.7-62.5)$ & $58.4(47.7-70.5)$ \\
\hline Spike & 215.8 (190.0-244.3) & $50.5(43.2-58.6)$ & $58.0(49.9-67.1)$ \\
\hline Receptor-binding domain & $295.1(260.3-333.6)$ & $72.1(62.0-83.3)$ & $81.3(70.2-93.6)$ \\
\hline \multicolumn{4}{|l|}{$\lg M$} \\
\hline Nucleocapsid proteins & $\mathrm{n} / \mathrm{a}$ & $\mathrm{n} / \mathrm{a}$ & $\mathrm{n} / \mathrm{a}$ \\
\hline Spike & $231.8(206.9-259.2)$ & $54.7(47.6-62.5)$ & $64.4(56.2-73.4)$ \\
\hline \multirow{2}{*}{\multicolumn{4}{|c|}{$\operatorname{lgG}$}} \\
\hline & & & \\
\hline Nucleocapsid proteins & 257.7 (222.6-297.0) & $55.8(43.4-70.0)$ & $55.4(44.4-68.1)$ \\
\hline Spike & $246.1(215.9-279.7)$ & $57.0(48.8-66.2)$ & $57.6(49.2-67.1)$ \\
\hline Receptor-binding domain & $408.3(364.0-457.3)$ & $101.0(88.4-114.8)$ & $97.3(85.1-110.8)$ \\
\hline
\end{tabular}

extremely low in both the WHO IS and CSCP HR and LR samples, which is the likely cause for the low predictive value of this analyte. ${ }^{10,11}$

A CSCP kit contains five DTS samples (blinded), $200 \mu \mathrm{L}$ PBS diluent, a 0.5-mL calibrated disposable micropipette, a dry silica pack, a printed DTS rehydration work aid, and a copy of the report form. The contents are sealed in a 1.5- $\times$ 9 -inch mailing tube. Users fill out a CSCP request form and each request is reviewed, assessed, and approved before the requested CSCP kit is shipped at ambient temperature with no cold packs. Users then use the kit according to their serology assay requirements. Users return their results and provide the information of the test platform used via electronic entry (CSCP Result Form) or e-mail a copy to COVIDPanel@ ucdenver.edu. A report decoding their samples is returned to the user; the report provides a comparison and interpretation of their results against the assigned value for the DTS samples they received. We provide a root-cause analysis to assist users in analyzing and determining corrective measures should the results provided be discordant with the assigned values.

As of June 2021, the CSCP has been shipped to multiple sites in Australia, Africa, Southeast Asia, North and South America, and Europe. CSCP concordance of the HR, NR, and LR samples are $97 \%, 93 \%$, and $65 \%$, respectively. We anticipated the LR sample would be a measure of the sensitivity of the test kit because of its construction or read-out method of the test platform; we are collecting these data for further analysis. Users have experienced a variety of logistical challenges, including receiving kits that were in transit for up to 3 months under harsh conditions $(n=1)$ and kits that were held for months before use $(n=2)$ without change in the expected results. One user reported issues with incomplete reconstitution and two users had problems uploading results to the website, all of which are under review for corrective actions.

Quality assurance is foundational for the validation of methods, external quality assurance, training, and inter-/ intra-laboratory comparison of serological tests. ${ }^{3,12-14}$ As global COVID-19 vaccination efforts are now underway, highly accurate and reliable SARS-CoV-2 serology testing is the primary method to assess vaccine efficacy. ${ }^{15}$ Many commercial and laboratory-developed tests react with a range of antigen targets, making it difficult to compare results in the absence of a common set of reference materials. To address this need, the CSCP was further evaluated against the WHO
IS using a microsphere immunoassay capable of measuring $\lg$, IgG, and total Ig reactivity to the $\mathrm{S}, \mathrm{RBD}$, and $\mathrm{N}$ antigens. By converting the CSCP HR and LR samples to the WHO IS BAUs, we provide the opportunity for direct interand intra-laboratory comparison of SARS-CoV-2 serological test results using calibrated reference samples. Widespread use of the CSCP for comparison of SARS-CoV-2 tests will help laboratories interpret and gain confidence in their results, while deterring laboratories from using poorly performing tests. In addition, the CSCP will help clinical laboratories inform their choice of diagnostic test to supplement clinical diagnoses of SARS-CoV-2 infection.

With this use in mind, our next step is to harmonize CSCP and other available serology reference materials by validating them concomitantly as secondary standards to the WHO IS. This would provide an inferential link to WHO IS and give broader access of validated reference materials to be used in comparing and evaluating test kit performance in use cases already cited. The DTS system is also flexible enough to accommodate additional samples to reflect current pandemic situations, such as post-vaccination and convalescent samples from persons infected with SARS-CoV-2 variants.

Received September 27, 2021. Accepted for publication November 9, 2021.

Published online January 7, 2022.

Note: Supplemental information is available at www.ajtmh.org.

Acknowledgments: We acknowledge the administrative assistance of Mary Moua and Linda S. Gabel. We are grateful to Susan Fink, Tara S. Givens, Suellen Nicholson, and Andy Schnaubelt for evaluating CSCP kits in their laboratories prior to kit release. We are thankful for the assistance of Karen E. Kulas.

Financial support: This project was funded by the Bill \& Melinda Gates Foundation (investment ID INV-006307 grant).

Disclosure: The acquisition of the plasma material was acquired through a Material Transfer Agreement between the University of Colorado and Vitalant (17.26.14).

Authors' addresses: William J. Windsor, Molly M. Lamb, and May C. Chu, Colorado School of Public Health, Anschutz Medical Campus, Aurora, CO, E-mails: wjwindsor58@yahoo.com, molly.lamb@cuanschutz.edu, and may.chu@cuanschutz.edu. Vijaya Knight, Patricia A. Merkel, and Leah Huey, Children's Hospital Colorado, Anschutz Medical Campus, Aurora, CO, E-mails: vijaya.knight@childrenscolorado.org, patricia. merkel@childrenscolorado.org, and leah.huey@childrenscolorado.org. Heidi R. Tucker, Kyle Carson, and Kelly M. Howard, Division of Infectious Diseases, Wadsworth Center, New York State Department 
of Health, Albany, NY, E-mails: heidi.tucker@health.ny.gov, kyle. carson@health.ny.gov, and kelly.howard@health.ny.gov. Jennifer L. Yates and William T. Lee, Division of Infectious Diseases, Wadsworth Center, New York State Department of Health, Albany, NY, and The School of Public Health, The University at Albany, Albany, NY, E-mails: jennifer.yates@health.ny.gov and william.lee@health.ny.gov. Mario L. Santiago, Mary K. McCarthy, Thomas E. Morrison, Ross M. Kedl, Ashley Frazer-Abel, Kejun Guo, Gillian Andersen, and Bradley S. Barrett, University of Colorado Anschutz Medical Campus, Aurora, CO, E-mails: mario.santiago@cuanschutz.edu, mary.mccarthy@cuan schutz.edu, thomas.morrison @ucdenver.edu, ross.kedl@cuansc hutz.edu, ashley.frazer-abel@cuanschutz.edu, kejun.guo@cuans chutz.edu, gillian.andersen@childrenscolorado.org, and bradley. barrett@cuanschutz.edu. Jessica M. Colón-Franco, Cleveland Clinic, Cleveland, OH, E-mail: colonj3@ccf.org.

This is an open-access article distributed under the terms of the Creative Commons Attribution (CC-BY) License, which permits unrestricted use, distribution, and reproduction in any medium, provided the original author and source are credited.

\section{REFERENCES}

1. Parekh BS et al., 2010. Dried tube specimens: a simple and cost-effective method for preparation of HIV proficiency testing panels and quality control materials for use in resourcelimited settings. J Virol Methods 163: 295-300.

2. Arora RK et al., 2021. SeroTracker: a global SARS-CoV-2 seroprevalence dashboard. Lancet Infect Dis 21: e75-e76.

3. Badrick T, Wienholt L, Fone D, Holzhauser D, 2020. The challenge of producing an EQA for the COVID-19 pandemic. Pract Lab Med 22: e00179.

4. DiGermanio CSG et al., 2021. SARS-CoV2 antibody persistence in COVID-19 convalescent plasma donors. MedRxiv.

5. Hasenkrug KJ et al., 2021. Recovery from acute SARS-CoV-2 infection and development of anamnestic immune responses in T cell-depleted rhesus macaques. bioRxiv.

6. EDI Epitope Diagnostics Inc., n.d. EDITM Novel Coronavirus COVID-19 ELISA Kits. Available at: http://www.epitop ediagnostics.com/covid-19-elisa. Accessed December 1, 2021.
7. Sabourin KR et al., 2021. Risk factors of SARS-CoV-2 antibodies in Arapahoe County first responders: the COVID-19 Arapahoe Serosurveillance Study (CASES) project. J Occup Environ Med 63: 191-198.

8. Schultz JS et al., 2021. Development and validation of a multiplex microsphere immunoassay using dried blood spots for SARS-CoV-2 seroprevalence: application in first responders in Colorado, USA. J Clin Microbiol 59.

9. Yates JL et al., 2021. Serological analysis reveals an imbalanced IgG subclass composition associated with COVID-19 disease severity. Cell Rep Med. 2: 100329.

10. National Institute for Biological Standards and Control, World Health Organization, 2020. WHO International Standard: First WHO International Standard for Anti-SARS-CoV-2 Immunoglobulin (Human): Instructions for Use. Available at: https:// www.nibsc.org/documents/ifu/20-136.pdf. Accessed December 1, 2021.

11. World Health Organization, 2020. Establishment of the WHO International Standard and Reference Panel for Anti-SARSCoV-2 Antibody. Available at: https://cdn.who.int/media/docs/ default-source/biologicals/ecbs/bs-2020-2403-sars-cov-2-ab-ik -17-nov-2020_4ef4fdae-e1ce-4ba7-b21a-d725c68b152b.pdf?s fvrsn=662b46ae_8\&download=true. Accessed December 1, 2021.

12. Klein $\mathrm{K}$ et al., 2020. A global proficiency testing programme for Xpert(R) MTB/RIF using dried tube specimens, 2013-2015. Afr J Lab Med 9: 1167.

13. Benzaken AS et al., 2014. External quality assurance with dried tube specimens (DTS) for point-of-care syphilis and HIV tests: experience in an indigenous populations screening programme in the Brazilian Amazon. Sex Transm Infect 90: 1418.

14. Beber AM, Sabido M, Vieira JM, Bazzo ML, Benzaken AS, 2015. External quality assessment in the voluntary counseling and testing centers in the Brazilian Amazon using dried tube specimens: results of an effectiveness evaluation. Rev Soc Bras Med Trop 48: 87-97.

15. West RGG, Kobokovich A, 2021. Variants, Vaccines and What They Mean for COVID-19 Testing. Available at: https:// www.jhsph.edu/covid-19/articles/variants-vaccines-and-whatthey-mean-for-covid19-testing.html. Accessed December 1, 2021. 